\title{
Efficacy of Narrow Band Imaging in Detecting Lower Gi Lesions and Its Histologic Correlation
}

\author{
Mohammad Moeen ${ }^{1}$, Afzal Anees ${ }^{1}$, Noora Saeed ${ }^{2}$ and Kafil Akhtar ${ }^{2}$ \\ ${ }^{1}$ Department of General Surgery, J.N. Medical College, Aligarh Muslim University, Aligarh. India \\ ${ }^{2}$ Department of Pathology, J.N. Medical College, Aligarh Muslim University, Aligarh. India
}

\section{ABSTRACT}

Background: To evaluate the efficacy of Narrow band imaging (NBI) in detecting lower GI lesions and differentiation on the basis of color, vascular pattern and surface pattern findings on NBI with histopathologic correlation.

Material and Methods: 94 patients of both gender, with lower GI symptoms were subjected for lower GI endoscopy by conventional white light endoscopy and CLV-190 HD colonoscope having Narrow-band imaging (NBI) as standard feature after obtaining an informed consent. Patients who did not give consent, patients with severe inflammatory bowel disease and who lost during follow up were excluded from the study. All NBI findings of suspected lesions were classified according to NICE (NBI International Colorectal Endoscopic) classification, into hyperplastic lesion, adenomatous and invasive carcinoma. Biopsies were taken and stained with Haematoxylin and Eosin as well as Periodic Acid Schiff stain. Statistical analysis was done to compare NBI diagnosis with the histopathological diagnosis.

Results: Majority of the patients were in the third decade of life, with a mean age of 36.22 years. Diarrhea was the commonest clinical presentation, in $60(63.8 \%)$ patients. NBI was suggestive of ulcerative colitis/Inflammatory bowel disease (IBD) in 59 (62.8\%) patients, neoplastic polypoidal lesion in 24 patients $(25.5 \%)$ and polypoidal non-neoplastic lesion in $11(11.7 \%)$ patients. Out of 59 (62.8\%) cases of Ulcerative colitis (UC), 35 (37.2\%) were polypoidal, among which 24 (68.6\%) were suggestive of neoplastic polypoidal lesion (NICE II-III) and $11(31.4 \%$ ) were non-neoplastic polypoidal lesion (NICE I). Out of 59 cases of UC, clear mucosal vascular pattern (MVP) was found in $11(18.6 \%)$ cases and obscure MVP in $48(81.4 \%)$ cases.

Conclusions: NBI provides a unique image with contrast enhancement and can emphasize the capillary pattern and surface architecture and plays a major role in the differentiation of neoplastic and non-neoplastic colorectal lesion, with high sensitivity and specificity.

Keywords: GI Lesions, Narrow Band Imaging, Endoscopy, Histopathology.

\section{Introduction}

Narrow band imaging (NBI) endoscopic technique enhances the mucosal morphology and vascularisation by applying an optical filter to the endoscopic light. ${ }^{[1]} \mathrm{NBI}$ basically works on the principle of depth of light penetration. Blue light is absorbed more superficially and green light is absorbed at a deeper layers. The resulting image is a combination of two independent absorption layers. The superficial capillaries will appear brown because the information of the blue is the input for both the green and blue channels. Deeper vessels will appear cyan because green is the input for both the red channel in the monitor.

Neoplastic changes are characterized by increased angiogenesis, and so adenomatous lesions typically appear darker due to increased microvessel density. ${ }^{[2]}$ This technique may improve the contrast between neoplastic and non-neoplastic tissue and hence could facilitate the detection as well as the differentiation of colonic lesions by just pushing button on scope handle. So NBI is also referred to as electronic or digital chromoendoscopy.
Chromoendoscopy and narrow band imaging have extensively been evaluated for their ability to detect colonic polyps on one hand, and for their diagnostic accuracy in differentiating neoplastic from non-neoplastic polyps on the other hand. Chromoendoscopy is a dye based technique that has been used more than two decades and has been shown to accurately differentiate between adenoma and hyperplastic polyps ${ }^{[3]}$ Most of the endoscopists are reluctant to adopt this technique for colorectal lesions screening, because it is a time consuming procedure and also has steep learning curve associated with interpretation of chromoendoscopic images.

The aim of this study was to see the efficacy of NBI in detecting lower GI lesions and differentiation on the basis of color, vascular pattern and surface pattern findings on NBI with histopathologic correlation.

\section{Material and Methods}

This prospective study was conducted in the Departments of Surgery and Pathology at Jawaharlal Nehru Medical College, Aligarh Muslim University, Aligarh, after taking 
the informed consent from the patients from July, 2014 to July, 2016. All patients included in the study underwent a thorough clinical workup (including detailed history, clinical examination and digital rectal examination.

The patients of both gender, with the lower GI symptoms were subjected for lower GI endoscopy by Olympus Evis Exera III CLV-190 HD colonoscope having Narrowband imaging (NBI) as standard feature after obtaining an informed consent. Patients who did not give consent, patients with severe inflammatory bowel disease and who lost during follow up were excluded from the study. Each suspected neoplastic lesions such as polyps or flat lesion was washed free of stool and mucus, and first examined by conventional white light endoscopy then by NBI colonoscopy. NBI yields a unique image that emphasizes the lesion color, microvascular architecture and surface pattern. All NBI findings of suspected lesions were classified according to NICE (NBI International Colorectal Endoscopic) classification, into hyperplastic lesion, adenomatous and invasive carcinoma. ${ }^{[4]}$ Biopsies were taken from all suspected lesions by punch tissue biopsy forceps, fixed in $10 \%$ formalin, paraffin embedded and cut into 3-5 $\mu$ thick sections and stained with Haematoxylin and Eosin as well as Periodic Acid Schiff-Diastase stain, when indicated. Finally, statistical analysis was done to compare the NBI diagnosis with the histopathological diagnosis.

\section{Results}

Majority of the patients were in the third decade of life, with a mean age of $36.22 \pm 16.9$ years.

Diarrhea was the commonest clinical presentation, present in $60(63.8 \%)$ patients, followed by bleeding per rectum in $58(61.7 \%)$, mucus with stool in $53(56.4 \%)$, pain abdomen in $48(51 \%)$.

Narrow Band Imaging on the lower GI lesions was performed on all 94 patients included in the study. It was suggestive of ulcerative colitis/Inflammatory Bowel Disease in $59(62.8 \%)$ patients, neoplastic polypoidal lesion in $24(25.5 \%)$ and polypoidal non-neoplastic lesion in $11(11.7 \%)$ patients. (Table 1) Fifty nine (62.8\%) cases were suspected of inflammatory lesion/UC, which was also the most common finding. Thirty five (37.2\%) cases were polypoidal, among which $11(31.4 \%)$ cases were suggestive of non-neoplastic polypoidal lesion on NBI (NICE I) (Figure 1) and 24 (68.6\%) cases were suggestive of neoplastic polypoidal lesion (NICE II-III) (Figure 2). (Table 2)

In a case of suspected ulcerative colitis (UC), assessment of signs of microscopic inflammation in acute and chronic form of the disease could be made on the basis of mucosal vascular pattern (MVP). Depending on the grade of inflammation, ulcerative colitis was divided into mucosal vascular pattern (MVP) - clear and obscure. Out of 59 cases of inflammatory colorectal lesion/UC, 48(81.4\%) cases had obscured MVP which was suggestive of mild form of UC. There were 11(18.6\%) cases of clear MVP on NBI endoscopy, which was suggestive quiescent phase of UC. (Table 3) On histopathological examination of 59 cases of UC, 39 patients were diagnosed as mild UC and 20 cases were diagnosed as quiescent UC (Figure 3).

On NBI examination of 35 cases of polypoidal colorectal lesions, 24 cases were provisionally diagnosed as neoplastic and 11 cases were suggestive of non- neoplastic lesions. Histopathological confirmation of above 35 lesions was done in the progressive part of the study. Out of 24 cases of suspected neoplastic polypoidal colorectal lesion on NBI, 22 cases were confirmed as malignant lesions on histopathology (Figure 4) and out of 11 suspected cases of non- neoplastic lesion on NBI, 9 were finally diagnosed as non-neoplastic polypoidal lesion on histopathology (Table 4). Of these 9 non neoplastic lesions, there were $6(66.7 \%)$ cases of hyperplastic polyps, $2(22.2 \%)$ cases of juvenile polyps and $1(11.4 \%)$ case of inflammatory polyp.

The sensitivity and specificity of NBI in differentiating between neoplastic and non-neoplastic colorectal lesion was $91.7 \%$ (CI-70.0\% to $98.8 \%$ ) and $81.8 \%$ (CI-48.2\% to 97.7\%) respectively, with PPV (positive predictive value) and NPV (negative predictive value) was 91.7\% (CI-73.0\% to $99.0 \%)$ and $81.8 \%(48.2 \%$ to $97.7 \%)$ respectively. Sensitivity and specificity of NBI in determining the grade of inflammation in UC was $100 \% 55.0 \%$ respectively. Positive predictive value and negative predictive value was $81.3 \%$ and $100 \%$ respectively.

Table 1: Lower GI endoscopic findings on NBI.

\begin{tabular}{|l|c|c|}
\hline Narrow band imaging findings & Number of cases & Percentage \\
\hline Ulcerative colitis /IBD & 59 & 62.8 \\
\hline Neoplastic lesions & 24 & 25.5 \\
\hline Non-neoplastic lesions & 11 & 11.7 \\
\hline Total & 94 & 100 \\
\hline
\end{tabular}


Table 2: NBI finding in neoplastic and non-neoplastic colorectal lesions.

\begin{tabular}{|l|c|c|}
\hline NBI findings & No. of patients & Percentage \\
\hline NICE I & 11 & 31.4 \\
\hline NICE II-III & 24 & 68.6 \\
\hline TOTAL & 35 & 100 \\
\hline
\end{tabular}

Table 3: NBI finding in inflammatory bowel disease/IBD.

\begin{tabular}{|l|c|c|c|c|}
\hline \multirow{2}{*}{$\begin{array}{l}\text { Mucosal Vascular } \\
\text { Pattern }\end{array}$} & Mild & Quiescent & N=59 \\
\cline { 2 - 5 } & 1 & 10 & 11 & 18.6 \\
\hline Clear & 32 & 16 & 48 & 81.4 \\
\hline
\end{tabular}

Table 4: Comparison between NBI finding and final histological finding in neoplastic and non-neoplastic (polypoidal) colorectal lesion.

\begin{tabular}{|l|c|c|c|}
\hline \multicolumn{2}{|l|}{} & \multicolumn{2}{c|}{ NBI Diagnosis } \\
\cline { 3 - 4 } & & Positive & Negative \\
\hline Neoplastic & 24 & $22(T P)$ & $02(F N)$ \\
\hline Non-neoplastic & 11 & $02(F P)$ & $09($ TN) \\
\hline Total & 35 & 24 & 11 \\
\hline
\end{tabular}

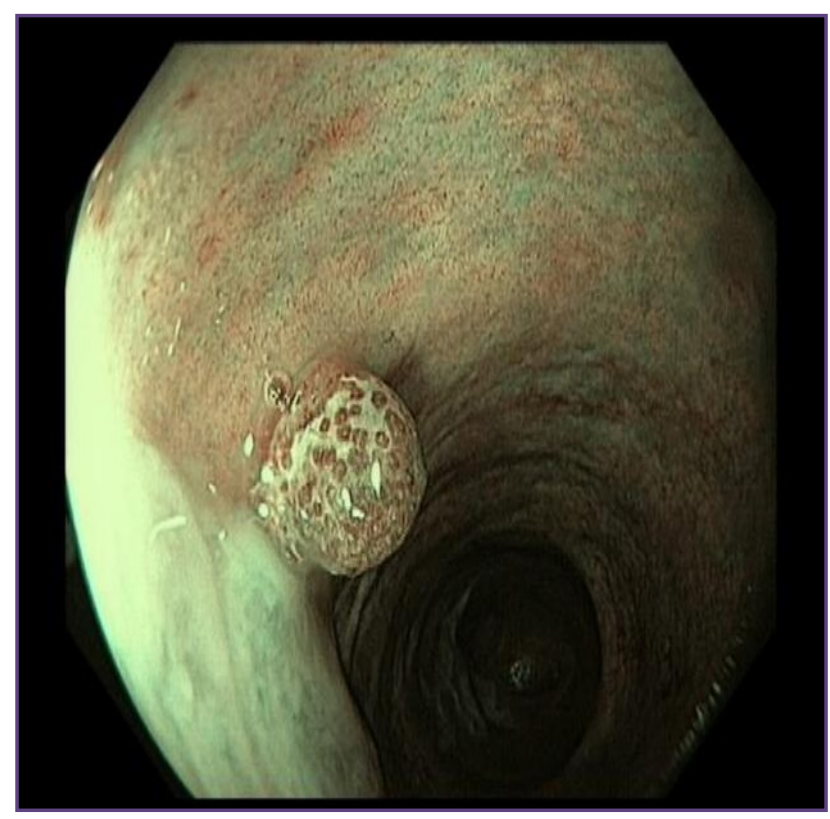

Fig. 1: Narrow band imaging lesion of hyperplastic polyp shows same color as background and multiple dark spots of uniform size present over the lesion (NICE I).

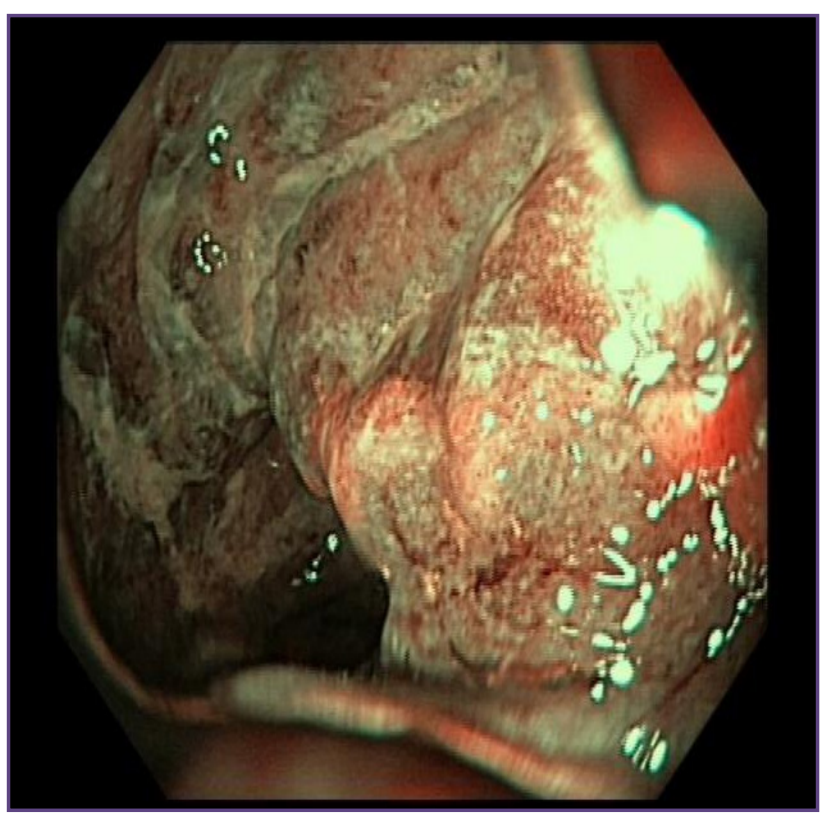

Fig. 2: Narrow band imaging of adenocarcinoma shows browner lesion relative to background and areas of markedly distorted pattern and vessels. 


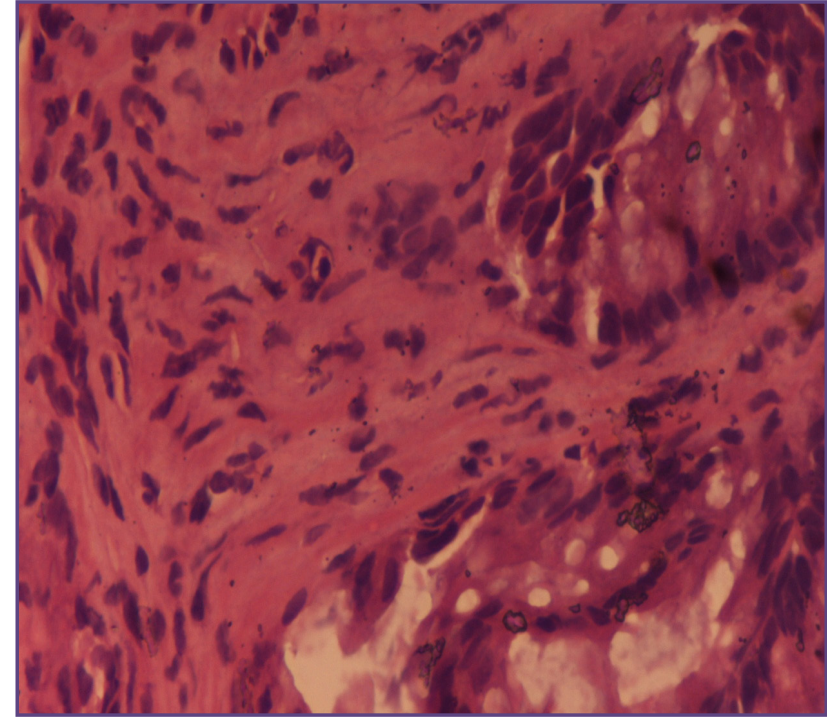

Fig. 3: Ulcerative Colitis (Quiescent form): Photomicrograph shows distorted mucosal glands surrounded by dense fibrous tissue proliferation (Haematoxylin and Eosin $x$ 40X).

\section{Discussion}

The principle of NBI is application of narrow band light filters for a detailed characterization of the vascular structures. Many studies have evaluated the role of NBI for the prediction of colorectal lesions (non-neoplastic and neoplastic) and compared it with chromoendoscopy or conventional endoscopy. ${ }^{[4,5]} \mathrm{NBI}$ was shown to have a higher accuracy than conventional colonoscopy, but similar to that of chromoendoscopy. ${ }^{[6,7]}$

On NBI endoscopy, in our study the most common findings was neoplastic polypoidal lesion (NICE II-III) and nonneoplastic polypoidal lesion (NICE I). Our findings were concordance with studies by Singh et al and Sano. ${ }^{[8,9]}$

Out of 59 inflammatory/UC colorectal lesions, 48 cases with obscure MVP were diagnosed as mild UC, and 11 cases of clear MVP were diagnosed as quiescent UC on NBI. In our study, the NBI vascular pattern in mild UC was obscure MVP and in quiescent UC was clear MVP, which was concordant with the studies of Kudo et al. ${ }^{[10]} \mathrm{We}$ found 39 lesions with mild UC and 20 with quiescent UC on histopathological examination.

Out of 35 polypoidal colorectal lesions, 24 cases of neoplastic lesion and 11 of non-neoplastic lesion were provisionally diagnosed on Narrow Band Imaging. Twentytwo out of 24 suspected cases of polypoidal neoplastic lesions and 9 out of 11 suspected cases of non-neoplastic lesions on NBI, were confirmed by histopathology. In our study, adenocarcinoma was the commonest colorectal

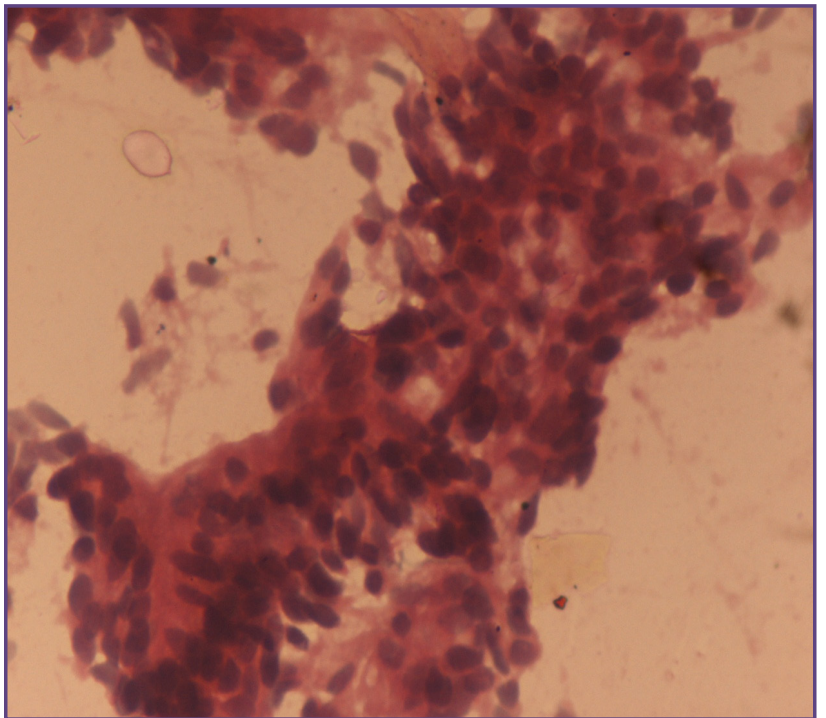

Fig. 4: Papillary carcinoma Colon: Imprint smear shows malignant cells in papillaroid configuration (Haematoxylin and Eosin $\mathrm{x}$ 40X).

malignant lesion and hyperplastic polyp was the commonest non-neoplastic colorectal lesion, findings concordant with that of Foss et al and Bond et al. ${ }^{[11,12]}$

In our study, the sensitivity and specificity of NBI in determining the grade of inflammation in UC was $100 \%$ (91.0\% to $100 \%)$ and $55.0 \%(31.5 \%$ to $76.9 \%)$ respectively. Our findings were similar to the reports of Kuznetsov and Matsumoto et al. ${ }^{[3,16]}$

The sensitivity and specificity of NBI in differentiating between neoplastic and non-neoplastic colorectal lesion was $91.7 \%$ (CI-70.0\% to $98.8 \%$ ) and $81.8 \%$ (CI-48.2\% to $97.7 \%)$ respectively. Our findings were concordant with the reports of Chiu, Machida et al, and Wu et al. ${ }^{[13-15]}$

\section{Conclusions}

NBI provides a unique image with contrast enhancement and can emphasize the capillary pattern and surface architecture and plays a major role in the differentiation of neoplastic and non-neoplastic colorectal lesion, with high sensitivity and specificity.

\section{References}

1. Su MY, Hsu CM, Ho YP, Chen PC, Lin CJ, Chiu CT. Comparative study of conventional colonoscopy, chromoendoscopy, and narrow-band imaging systems in differential diagnosis of neoplastic and nonneoplastic colonic polyps. Am J Gastroenterol 2006; 101:2711-16.

2. Lapalus MG, Helbert T, Napoleon B, Rey JF, Houcke P. Does chromoendoscopy with structure enhancement improve the colonoscopic adenoma detection rate? Endoscopy 2006; $38: 444-48$ 
3. Kuznetsov K, Lambert R, Rey JF. Narrow-band imaging: Potential and limitation. Endoscopy 2006; 38:76-81.

4. Konerding MA, Fait E, Gaumann A. 3D microvascular architecture of pre-cancerous lesion and invasive carcinoma of the colon. Br J Cancer 2001; 84:1354-62.

5. East JE. Narrow band imaging with magnification for the characterization of small and diminutive colonic polyps: pit pattern and vascular pattern intensity, Endoscopy 2008; 40:811-17.

6. Rastogi A, Pondugula K, Bansal A. Recognition of surface mucosal and vascular patterns of colon polyps by using narrow-band imaging: interobserver and intraobserver agreement and prediction of polyp histology. Gastrointest Endosc 2009; 69(32):716-22.

7. East JE, Kamm MA, Bassett P, Saunders BP. Magnification panchromo-endoscopy remains the mainstay for colitis dysplasia detection and differentiation. Gastroenterol 2007; 133: 366-67.

8. Singh R, Anagnostopoulos G, Yao K, Karageorgiou H, Fortun $\mathrm{P}$, ShondeA et al. Narrow-band imaging with magnication in barrett's esophagus: validation of a simplified grading system of mucosal morphology patterns against histology. Endoscopy 2008; 40(6): 457-63.

9. Sano Y. Narrow Band Imaging (NBI). Clin Gastroenterol 2009; 24: 47-52.
10. Kudo T, Matsumoto T, Esaki M, Yao T, Iid M. Mucosal vascular pattern in ulcerative colitis: observations using narrow band imaging colonoscopy with special reference to histologic inflammation. Int J Colorectal Dis 2009; 24:495-99.

11. Foss F, West K, McGregor A. Pathology of polyps detected in the bowel cancer screening programme. Diagn Histopathol 2011; 17:495-99.

12. Bond JH. Polyp guideline: diagnosis, treatment, and surveillance for patients with colorectal polyps. Practice Parameters Committee of the American College of Gastroentrology. Am J Gastroenterol 2000; 95: 3053-63.

13. Chiu HM, Chang CY, Chen CC. A prospective comparative study of narrow-band imaging, chromoendoscopy and conventional colonoscopy in the diagnosis of colorectal neoplasia. Gut 2007; 56:373-79.

14. Machida H, Sano Y, Hamamoto Y, Muto M, Kozu T, Tajiri H, Yoshida S. Narrow-band imaging in the diagnosis of colorectal mucosal lesions: a pilot study. Endoscopy 2004; 36:1094-98.

15. Wu L, Li Y, Li Z, Cao Y, Gao F. Diagnostic accuracy of narrow- band imaging for the differentiation of neoplastic from non- neoplastic colorectal polyps: a meta-analysis. Colorectal Dis 2013; 15:3-11.

16. Matsumoto T, Kudo T, Yao T, lida M. Auto fluorescence imaging colonoscopy in ulcerative colitis: comparison with conventional and narrow-band imaging colonoscopy. Diag Endosc 2010; 21:139-43.

*Corresponding author:

Dr. Kafil Akhtar, Professor, Department of Pathology, Jawaharlal Nehru Medical College, Aligarh Muslim University, Aligarh.(U.P)-India. Email: drkafilakhtar@gmail.com

Financial or other Competing Interests: None. 